\title{
Macular pucker, an atypical clinical presentation of ocular toxoplasmosis: a case report
}

\author{
Si Zhang ${ }^{1 \dagger}$, Chun-yan Xue ${ }^{2 \dagger}$, Ya-jun Liu', Wen-wen Zhang ${ }^{1}$ and Zheng-gao Xie ${ }^{1 *}$ (D)
}

\begin{abstract}
Background: Ocular toxoplasmosis caused by Toxoplasma gondii is an infectious disease which is widely distributed around the world and can present with various clinic manifestations. We are here reporting an unusual case presented with epiretinal membrane (ERM), i.e., macular pucker.

Case presentation: A 16-year old male patient visited our outpatient clinic complaining of decreased vision for about 8 years in his left eye. The best-corrected visual acuity (BCVA) was 20/20 OD and 20/400 OS. There was sensory exotropia in his left eye. No inflammatory cells or flare were found in his anterior chamber or vitreous cavity OU. An ERM involving his left macular area was found on his dilated fundus exam, which was confirmed by Optical Coherence Tomography (OCT). The ERM was found to involve his left macular area with his foveal ellipsoid zone absent. The right eye was found to be within normal limit.

After a thorough discussion with the patient and his parents about treatment options and surgical benefits, risks and alternatives, we performed vitrectomy, peeled off the ERM and collected the vitreous sample for parasite testing during the procedure. Patient's blood also was drawn for serological testing. Vitreous sample analysis and serological tests confirmed ocular toxoplasmosis OS as his final diagnosis. Unfortunately, the BCVA of this patient was not improved after the surgery, but the exotropia disappeared.
\end{abstract}

Conclusion: ERM is an unusual clinical presentation of ocular toxoplasmosis. We may add Toxoplasma gondii infection as a differential diagnosis when encountering ERM cases.

Keywords: Epiretinal membrane, Macular pucker, Ocular toxoplasmosis, Toxoplasma gondii, Intraocular fluid testing, Serologic testing

\section{Background}

Toxoplasmosis is an infectious disease caused by the obligate intracellular parasite Toxoplasma gondii, which is widely distributed around the world. About $30 \%$ of people in the world are infected with toxoplasmosis [1]. Cats are the only definitive host, while human and other

\footnotetext{
* Correspondence: zgxie87@163.com

${ }^{\dagger}$ Si Zhang and Chun-yan Xue contributed equally to this work. 'Department of Ophthalmology, Nanjing Drum Tower Hospital, The Affiliated Hospital of Nanjing University Medical School, Nanjing 210008, Jiangsu Province, China

Full list of author information is available at the end of the article
}

animals are the intermediate hosts. The transmission routes of Toxoplasma include ingestion of food and water contaminated by cysts, blood transfusion, organ transplantations and transplacental transmission [2]. Patients with active disease frequently present with retinochoroidal lesions. Active toxoplasmic retinochoroiditis most often involves the posterior pole (76\%), and is more common found in the unilateral eyes. More than $90 \%$ of patients have normal visual acuity in the contralateral eye [3]. Ocular toxoplasmosis can cause various complications, including exudative retinal detachment, retinal vascular occlusion, choroidal neovascularization,

(c) The Author(s). 2021 Open Access This article is licensed under a Creative Commons Attribution 4.0 International License, which permits use, sharing, adaptation, distribution and reproduction in any medium or format, as long as you give appropriate credit to the original author(s) and the source, provide a link to the Creative Commons licence, and indicate if changes were made. The images or other third party material in this article are included in the article's Creative Commons licence, unless indicated otherwise in a credit line to the material. If material is not included in the article's Creative Commons licence and your intended use is not permitted by statutory regulation or exceeds the permitted use, you will need to obtain permission directly from the copyright holder. To view a copy of this licence, visit http://creativecommons.org/licenses/by/4.0/ The Creative Commons Public Domain Dedication waiver (http://creativecommons.org/publicdomain/zero/1.0/) applies to the data made available in this article, unless otherwise stated in a credit line to the data. 
pre-retinal membrane, macular hole and macular edema [4-7]. Our case showed an atypical manifestation, a white oval-shaped ERM in the involved eye. We reviewed all cases of ocular toxoplasmosis in the literature, secondary ERM as the main presentation has not yet been reported. We are reporting this rare case as reminding for considering the possibility of Toxoplasma gondii infection for ERM in adolescent patients.

\section{Case presentation}

A 16-year old male patient visited our outpatient clinic with a chief complaint of decreased vision for about 8 years in his left eye. He had a history of close contact with cats and dogs, with no history of any other systemic illness, trauma or surgery. The best-corrected visual acuity (BCVA) was 20/20 in his right eye and 20/400 in his left eye. The intraocular pressure was normal in both eyes. There was sensory exotropia in his left eye. No inflammatory cells were found in his anterior chamber or vitreous OU. The fundus exam of his left eye showed a white oval-shaped ERM with the size of of 2.0 papillary diameter, some pigmented chorioretinal scars, and yellow-white round retinochoroidal lesions on the temporal side of the macula (Fig. 1a). Spectral-domain optical coherence tomography (SD-OCT) confirmed a thickened ERM with vitreous retinal traction involving the macula, and ellipsoid zone defects in the central macula (Fig. 1b).

A preliminary diagnosis of parasitic infection was made based on the choroidoretinopathy in the posterior pole of his left eye. To further confirm this diagnosis, $2.0 \mathrm{ml}$ of vitreous humor was collected during the following vitrectomy and sent for parasite testing. Meanwhile, his blood sample was also collected and sent for serological testing. During the surgery, the ERM was peeled off with caution, together with a brown pigmented lesion attached beneath (Fig. 1c). The proliferative membranes were sent for histopathological examination, which later on reported "hyalinosis of local organization found in the samples" (Fig. 1d). Vitreous sample analysis found Toxoplasma DNA undetectable, but Toxoplasma IgG to be $6.95 \mathrm{IU} / \mathrm{ml}$ (reference range less than $4 \mathrm{IU} / \mathrm{ml}$ ). His serum Toxoplasma IgG was found to be $20 \mathrm{IU} / \mathrm{ml}$ (reference range was less than $20 \mathrm{IU} / \mathrm{ml}$ ). The Goldmann-Witmer coefficient (GWC) value of Toxoplasma gondii was 36.72 (reference range 0 2). Based on those test results listed above, the final diagnose was ocular toxoplasmosis involving his left eye.

Unfortunately, 1 month after the surgery, the patient's BCVA was still 20/400 (no effect of correction). His intraocular pressure was still within normal limits.

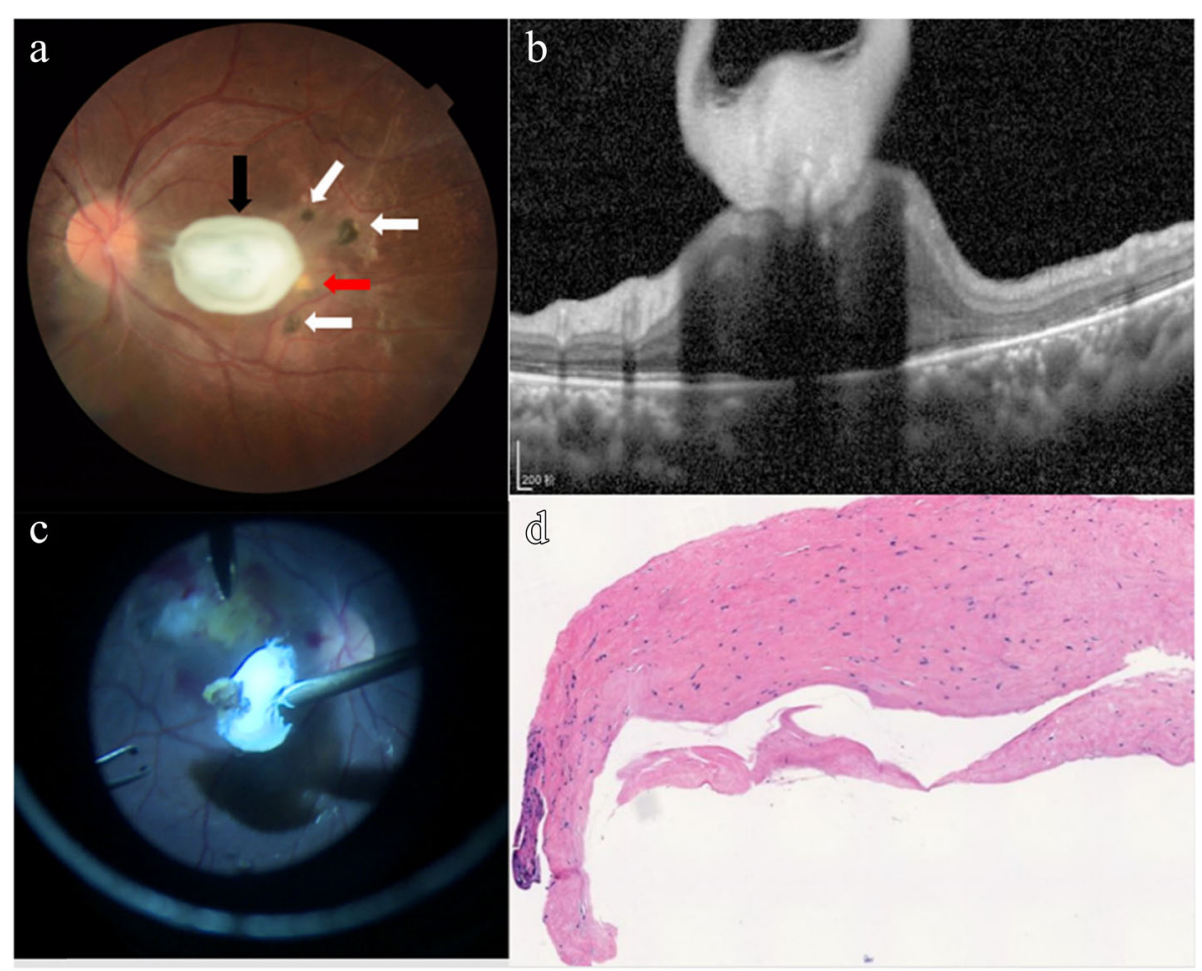

Fig. 1 Fundus photograph. A white oval-shaped thickened ERM (black arrow), pigmented chorioretinal scars (white arrows) and yellow-white round retinochoroidal lesions (red arrow) temporal to macula (a). Spectral-domain optical coherence tomography (SD-OCT). ERM and traction involving the macula, ellipsoid zone defects in the central macula (b). Intraoperative findings. ERM was peeled off, a brown pigmented lesion attached beneath (c). Histopathological examination of the ERM, hyalinosis of local organization found in the samples (HEx 100) (d) 
Unexpectedly, his left exotropia resolved after the surgery. The lens and vitreous remained unremarkable. Postoperative fundus examination (Fig. 2a) revealed no obvious changes of the pigmented chorioretinal scars and yellow-white retinochoroidal lesions on the temporal side of the macula. A repeat SD-OCT showed a reduction in central macular thickness (Fig. 2b).

\section{Discussions and conclusions}

In this case, the patient came from a rural area, with a history of cats and dogs close contact, and a habit of drinking cold unboiled tap water and eating raw vegetables since his childhood. These all put him at risk for toxoplasmosis infection. Several pigmented chorioretinal scars and yellow-white retinochoroidal lesions were found in the posterior pole of the left eye, suggested the possibility of ocular toxoplasmosis, even though there were no inflammatory cells found in the anterior chamber and vitreous cavity. The preliminary diagnosis was later on confirmed by further serologic and vitreous analysis.

Toxoplasma gondii transmission can be congenital (transplacental) or postnatally acquired [8]. Congenital infection refers to the transmission of Toxoplasma to the fetus through the placenta by pregnant women suffering from acute toxoplasmosis or reinfection with toxoplasmosis. Children are mostly asymptomatic at birth and may later on develop visual loss, nystagmus, sensory exotropia, and neurological symptoms, such as cerebral edema, brain calcification, and/or intellectual disability. Our patient reported here had normal visual acuity in both eyes and nervous system development since childhood reported by his parents and presented with a loss of vision at the age of eight, so congenital Toxoplasma gondii infection could be excluded. Acquired Toxoplasma gondii infection in children is often difficult to diagnose, because of the absence of accurate and timely complaints due to patients' young age. Therefore, there is great difficulty in early diagnosis and treatment of uveitis. If early diagnosis and treatment are not carried out, it may cause severe visual impairment [9]. In this case, the visual prognosis for the patient was poor due to macular destruction, possible amblyopia and sensory exotropia. Ocular toxoplasmosis is the most common type of pediatric posterior uveitis, accounting for up to $50 \%$ of posterior uveitis cases [10]. ERM secondary to uveitis is common, but ERM secondary to ocular toxoplasmosis as the main presentation has not been reported. The formation of the ERM is due to the inflammatory response that causes retinal pigment epithelium (RPE) cells, glial cells, Müller cells, fibroblasts to migrate and proliferate and secrete extracellular matrix [11]. Miranda [12] reported 14 patients with macular ERM secondary to ocular toxoplasmosis, all of whom had a relatively transparent and thin type of ERM which were completely different from the white dense thick ERM found in our case. All patients underwent vitrectomy combined with ERM peeling. The average time from diagnosis to surgery was $36.57 \pm 24.40$ months. The results showed that 12 patients presented with different degrees of improved visual acuity, and 2 patients had no improvement in visual acuity. Adan [13] reported 2 patients with ERM secondary to ocular toxoplasmosis who underwent vitrectomy combined with ERM peeling. Postoperative SD-OCT showed that the macular foveal traction was relieved, and both patients had visual acuity improvement without postoperative complications.

In our case, the ERM covering the macular area of the patient's left eye was accompanied by severe visual loss with sensory exotropia. After thorough preoperative examinations and detailed discussions of benefits, risks and alternatives with the patient and his parents, a middle and posterior vitrectomy combined with ERM peeling surgery was performed. One month after the surgery, the patient's BCVA was still 20/400, but the exotropia vanished and the eye position was notably improved.

Currently, intraocular fluid and serologic testing of Toxoplasma gondii DNA, IgG and IgM are commonly used to diagnose Toxoplasma gondii infection. The patient's vitreous humor and blood specimens were tested for Toxoplasma DNA, IgG and IgM. The results of

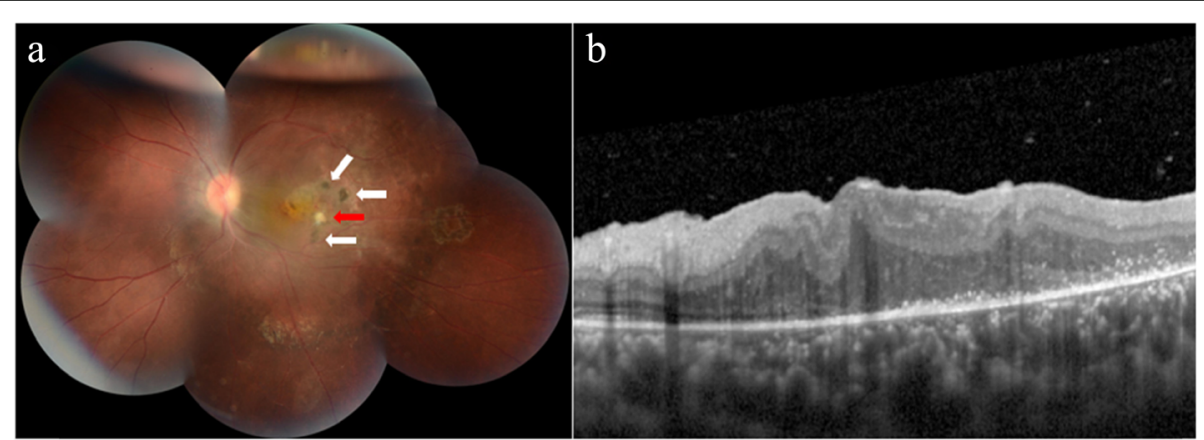

Fig. 2 Fundus photography after surgery and SD-OCT scan of the patient. The pigmented chorioretinal scars (white arrows) and yellow-white retinochoroidal lesions (red arrow) at temporal macula had no obvious changes (a). Reduction in central macular thickness (b) 
intraocular fluid showed that Toxoplasma DNA was zero, Toxoplasma IgG was $6.95 \mathrm{IU} / \mathrm{ml}$ (reference range less than $4 \mathrm{IU} / \mathrm{ml})$. His serum Toxoplasma IgG was 20 $\mathrm{IU} / \mathrm{ml}$ (reference range was less than $20 \mathrm{IU} / \mathrm{ml}$ ), and the GWC value of Toxoplasma was 36.72 (reference range 0 $\sim 2$ ). Because Toxoplasma gondii is an obligate intracellular parasite, the positive rate of Toxoplasma DNA detection in intraocular fluid is usually low, about $30-40 \%$ [14]. Although Toxoplasma DNA in the intraocular fluid of this patient was zero, Toxoplasma gondii infection was diagnosed when the GWC value was found by over four.

The classical treatment of ocular toxoplasmosis is triple therapy with pyrimethamine, sulfadiazine and prednisone [2]. For immunocompetent patients, toxoplasmic chorioretinitis is a self-limiting inflammation that resolves spontaneously within $4-8$ weeks. Since no drug has been found to cure the infection in human hosts, the goal of antimicrobial treatment continues to be limiting parasite multiplication during active retinitis [15]. In this case, the patient with inactive ocular toxoplasmosis received no antimicrobial treatment after surgery considering the side effects of antiparasitic drugs. The patient was followed up 4 months after the surgery, his left eye was still in stable condition with the absence of active inflammation.

ERM, especially thick ERM, secondary to ocular toxoplasmosis as the main presentation is rare. Intraocular fluid and serologic testing can provide sufficient evidence for the diagnosis of toxoplasmosis. Vitrectomy combined with ERM peeling is an effective treatment for ERM secondary to ocular toxoplasmosis. This case serves as a reminder that the possibility of Toxoplasma gondii infection should be taken into consideration in pediatric ERM patients.

\section{Abbreviations}

ERM: Epiretinal membrane; BCVA: Best-corrected visual acuity; SDOCT: Spectral-domain optical coherence tomography; RPE: Retinal pigment epithelium; GWC: Goldmann-Witmer coefficient

\section{Acknowledgements}

Not applicable.

\section{Authors' contributions}

SZ, CYX, YJL and WWZ collected the data, SZ and WWZ wrote the first draft. ZGX Revised the draft with important intellectual content. All authors

approved the final version. All authors agree to be accountable for the work.

\section{Funding}

Not applicable.

\section{Availability of data and materials}

All data supporting the conclusions of this article are included in the present article.

\section{Declarations}

Ethics approval and consent to participate Not applicable.

\section{Consent for publication}

Written informed consent was obtained from the patient's parents for publication of this case report and any accompanying images (no identifying patient data). A copy of the written consent is available for review by the editor of this journal.

\section{Competing interests}

The authors declare that they have no competing interests.

\section{Author details}

'Department of Ophthalmology, Nanjing Drum Tower Hospital, The Affiliated Hospital of Nanjing University Medical School, Nanjing 210008, Jiangsu Province, China. ${ }^{2}$ Department of Ophthalmology, Jinling Hospital, Nanjing University School of Medicine, Nanjing 210008, Jiangsu Province, China.

Received: 13 January 2021 Accepted: 10 May 2021

Published online: 17 May 2021

\section{References}

1. Garza-Leon M, Garcia LA. Ocular toxoplasmosis: clinical characteristics in pediatric patients. Ocul Immunol Inflamm. 2012;20(2):130-8.

2. Montoya JG, Liesenfeld O. Toxoplasmosis. Lancet. 2004:363(9425):1965-76.

3. Chan NS, Choi J, Cheung CMG, et al. Pediatric uveitis. Asia Pac J Ophthalmol. 2018;7(3):192-9.

4. Holland GN. Ocular toxoplasmosis: a global reassessment. Part II: disease manifestations and management. Am J Ophthalmol. 2004;137(1):1-17.

5. Rodríguez Á, Gómez FE, Valencia M, et al. Peripheral retinal neovascularization in recurrent cicatricial toxoplasmic retinochoroiditis: case series report. Eur J Ophthalmol. 2015;25(2):159-62

6. Arana B, Fonollosa A, Artaraz J, et al. Macular hole secondary to toxoplasmic retinochoroiditis. Int Ophthalmol. 2014:34(1):141-3.

7. Papadopoulou DN, Petropoulos IK, Mangioris $\mathrm{G}$, et al. Pars plana vitrectomy in the treatment of severe complicated toxoplasmic retinochoroiditis. Eur J Ophthalmol. 2011;21(1):83-8

8. Garza-Leon M, Muccioli C, Arellanes-Garcia L. Toxoplasmosis in pediatric patients. Int Ophthalmol Clin. 2008;48(3):75-85.

9. Lonngi M, Aguilar MC, Ríos HA, et al. Pediatric uveitis: experience in Colombia. Ocul Immunol Inflamm. 2016;24(4):410-4.

10. Patel H, Goldstein D, FRCSC. Pediatric uveitis. Pediatr Clin N Am. 2003;50(1): 125-36.

11. Raval V, Rao S, Das T. Anatomical and functional outcomes of pars plana vitrectomy for inflammatory epiretinal membrane surgery in healed toxoplasmosis infection. Indian J Ophthalmol. 2018;66(10):1485-9.

12. Miranda AF. Costa de Andrade G, Novais EA, et al. outcomes after pars plana vitrectomy for epiretinal membranes associated with toxoplasmosis. Retina 2016:36(9):1713-7.

13. Adan A, Giralt J, Alvarez G, et al. Pars plana vitrectomy forvitreoretinal complications of ocular toxoplasmosis. Eur J Ophthalmol. 2009;19(6):1039-43.

14. Santos FF, Nascimento $H$, Muccioli C, et al. Detection of toxoplasma gondii DNA in peripheral blood and aqueous humor of patients with Toxoplasmic active focal necrotizing retinochoroiditis using real-time PCR. Arq Bras Oftalmol. 2015;78(6):356-8.

15. Butler NJ, Furtado JM, Winthrop KL, Smith JR. Ocular toxoplasmosis II: clinical features, pathology and management. Clin Exp Ophthalmol. 2013; 41(1):95-108.

\section{Publisher's Note}

Springer Nature remains neutral with regard to jurisdictional claims in published maps and institutional affiliations. 